\title{
Tempo discrimination of musical patterns: Effects due to pitch and rhythmic structure
}

\author{
MARILYN G. BOLTZ \\ Haverford College, Haverford, Pennsylvania
}

\begin{abstract}
The purpose of this research was to investigate a set of factors that may influence the perceived rate of an auditory event. In a paired-comparison task, subjects were presented with a set of music-like patterns that differed in their relative number of contour changes and in the magnitude of pitch skips (Experiment 1) as well as in the compatibility of rhythmic accent structure with the arrangement of pitch relations (Experiment 2). Results indicated that, relative to their standard referents, comparison melodies were judged to unfold more slowly when they displayed more changes in pitch direction, greater pitch distances, and an incompatible rhythmic accent structure. These findings are suggested to stem from an imputed velocity hypothesis, in which people overgeneralize certain invariant relations that typically occur between melodic and temporal accent structure within Western music.
\end{abstract}

In the study of event perception, one issue of particular interest is the effects of an event's spatiotemporal structure on cognitive behavior. Any given event, whether it is a melody, speech utterance, or visual scene, is necessarily dynamic in nature and displays an array of nontemporal (spatial) information that unfolds with a given rate and rhythmicity over a total time span. In considering how this information is processed by the cognitive system, several studies have examined the effects of an event's rhythm, relative to the array of nontemporal information, on perception and selective attending skills as well as the subsequent remembering of events (see Fraisse, 1978, 1982, for reviews). Others have focused on the experienced and remembered duration of an event and on how characteristics of the information filling a time span influence both the accuracy and the bias of judgments (see Allan, 1979; Block, 1979; Fraisse, 1984, 1987, for reviews). Surprisingly, however, there is relatively little research in which those factors that influence the perception and remembering of tempo information are investigated. This omission in the literature is particularly puzzling in that the rate of informational flow will directly determine an event's total duration and has been found to alter the perceived rhythm of an event (Clarke, 1982; Handel, 1993; Marshburn \& Jones, 1985; Pierson, 1976; Povel, 1977). Hence, tempo is a primary aspect of an event's structure that covaries with other temporal dimensions and, therefore, merits additional investigation, in order to determine its role in cognitive behavior.

The purpose of the present research is to investigate a set of variables that may influence the perceived tempo of music-like patterns. One such variable involves the po-

This research was supported by a Faculty Research Grant from Haverford College. The author thanks Gabriel Dichter and Erika Wilde for the collection of data, as well as Robert Remez, Geoff Collier, and Stephen Handel for their critical comments on this manuscript. All correspondence should be addressed to M. Boltz, Department of Psychology, Haverford College, Haverford, PA 19041 (e-mail: mboltz@haverford.edu). tential impact of a melody's rhythm and whether the arrangement of temporal accents, relative to the array of melodic structure, can alter the apparent rate of a tune. With the second set of factors, the role of pitch information and how the overall contour of pitch intervals (i.e., the ups and downs of pitch), as well as the magnitude of pitch skips, may also exert a significant impact on behavior are addressed. This, in turn, leads one to consider the relationship between a melody's temporal and its nontemporal information and how these two aspects of event structure jointly influence one another.

\section{Effects of Musical Tempo on Cognitive Behavior}

In general, much of the past research on event tempo has been conducted in the area of music cognition. Tempo is considered not only to be a primary means of aesthetic expression and of inducing certain moods within an audience but also to be a determinant of both the accuracy and the precision with which a composition is performed. In reviewing the literature, it is clear that much of this research has focused on three aspects of cognitive behavior.

The first aspect concerns musical preference and whether people find some tempi more enjoyable to listen to than others. Across several different studies, the general finding is that subjects of different age groups prefer faster melodies, including those compositions that have been speeded up relative to their unaltered versions (Geringer, 1976; LeBlanc, 1981; LeBlanc \& McCrary, 1983). Listener preference, however, also depends on one's internal tempo and preferred rate of activity (i.e., preferred rate of tapping, walking, speaking, etc.). For example, individuals who have been clinically diagnosed as manic tend to prefer faster paced music than do normal control subjects and to prefer tempi that closely coincide with their own preferred tapping rate (Nielzen \& Cesarec, 1982; Stein, 1977).

In a second area of research, the ability to remember musical tempi over prolonged time periods has been in- 
vestigated. Overall, analyses of memory performance indicate that people are remarkably skilled. For example, when one compares the tempi of compositions played over multiple occasions by a given performing group, the degree of variability is extremely low and approximates a value of $0.2 \%$ (Clynes \& Walker, 1982, 1986; Collier \& Collier, 1994). Nonmusicians display a similar pattern of results when asked to sing, hum, or whistle popular rockand-roll tunes from memory (Levitin \& Cook, 1996). The correlation of subjects' productions with the songs' actual tempi is .95 , and $72 \%$ of all subjects yield an average variance of $\pm 4 \%$. When errors occur, people are more apt to sing too fast than to sing too slowly, a bias that has been found in other production tasks (Kuhn, 1977).

The third area of research is of most interest to the present set of studies and involves people's ability to detect any changes in a melody's tempo. One primary issue concerns the assessment of how much of a change in tempo is necessary in order to detect a just noticeable difference (JND) for tempo. Using a two-alternative forced choice technique ("which sequence is faster?"), Drake and Botte (1993) found that temporal thresholds are directly dependent on the number of intervals within a sequence: For single intervals, the average JND is around $6 \%$ and gradually decreases to $3 \%$ with six-tone intervals. Across all sequences, the optimal sensitivity was observed for those interonset intervals ranging between 300 and $800 \mathrm{msec}$. Others have investigated whether tempo changes in one direction are more easily detected than changes in the other direction. Although several studies have found that decreases in tempo are more readily discriminable than increases (Kuhn, 1974; Madsen, 1979), others have found the reverse (Geringer \& Madsen, 1984; Wang \& Salzberg, 1984; Yarbrough, 1987). Lastly, Wapnick (1980) has examined people's sensitivity to tempo variations that span over a greater range than that studied in past research. In this study, subjects listened to two versions of the same musical excerpt, one being between $60 \%$ and $169 \%$ faster or slower than the other. The subjects' task on each trial was to judge the second excerpt, relative to the first, using a magnitude estimate. Results showed a systematic pattern of bias: Tempo deviations were overestimated in their magnitude when the change in the comparison pattern was less than twice that of the standard but underestimated in magnitude when the change was more than twice that of the standard. As a set, then, these studies suggest that tempo discrimination is quite accurate within a restricted range but becomes imprecise when this range is exceeded.

\section{The Potential Impact of Pitch Relations}

To date, much of the past research on tempo discrimination has focused on issues related to Weber's Law and has, therefore, held all aspects of musical structure constant while systematically manipulating tempo variations. This research, in turn, is very useful, because it allows one to determine those psychophysical principles that apply to tempo perception alone. However, the overall generality of these data is unknown, because the potential impact of other structural dimensions has rarely been addressed. Musical compositions display a highly complex array of information in which pitch intervals can change in direction, bear different relationships to a melody's underlying tonality scheme, move from one timbre to another, and unfold within a given rhythmic context. All of these factors may influence the perceived rate of a melody and exert a systematic impact on a wide variety of cognitive behaviors, including those of selective attending and remembering skills as well as listeners' aesthetic judgments. Hence, in order to fully understand melodic processing, it is necessary to examine the effects of tempo in conjunction with these other aspects of musical structure.

From a theoretical perspective, the potential influence of a melody's nontemporal information (i.e. pitch characteristics) on the perception of temporal information gives rise to at least two questions of interest. The first concerns whether these two structural dimensions are independent or interactive in nature. This issue is one that has arisen in other areas of musical cognition in which the relationship between pitch and rhythm, in particular, has been a subject of debate. According to one perspective, pitch and rhythm are processed independently, so that the influence of one dimension does not depend on the characteristics of the other (Palmer \& Krumhansl, 1987). For example, certain types of rhythms may facilitate attending and remembering, regardless of the accompanying pitch pattern. Evidence for this type of relationship primarily occurs when subjects are asked to judge the similarity of different melodies (Monahan \& Carterette, $1985)$ or to rate melodies on different perceptual dimensions (Palmer \& Krumhansl, 1987). In contrast, others have argued that pitch and rhythm exert an interactive influence on behavior in which the effect of one dimension is dependent on the other (Boltz \& Jones, 1986; Jones \& Boltz, 1989). That is, a given rhythm may facilitate the perception and remembering of some pitch patterns but not others. This type of relationship has, in fact, been found in the expectancy generation of musical relations (Boltz, 1989, 1993; Schmuckler \& Boltz, 1994) as well as in the recall and recognition of pitch (Boltz, 1991a; Boltz \& Jones, 1986; Jones, Boltz, \& Kidd, 1982) and of durational information (Boltz, 1992, 1994, 1995, 1998). In sum, then, there is evidence for both views, and the overall issue remains unresolved.

Some insight, however, may come from examining the effects of pitch relations on tempo perception. Judgments of a melody's overall rate may be independent of the accompanying melodic structure of a tune or, alternatively, may reveal an interactive relationship in which tempo is perceived to alter as a function of pitch information. This, in turn, may indicate what types of contexts influence the 
cognitive processing of temporal and nontemporal information and how each dimension is encoded relative to the other.

If pitch and tempo are found to be dependent on one another, this raises a second question of interest—namely, what types of pitch relationships result in an acceleration or deceleration of perceived tempo, and what mediational mechanisms are responsible for this behavior? To date, there is no theoretical model that addresses the potential interaction between pitch and tempo information. One conceptual framework, however, may stem from certain structural relations between melodic and temporal accent structures that are used by composers to highlight a melody's organization to listeners. Accent structures have been found to influence a wide variety of cognitive behaviors, and these effects may extend to the perception of auditory motion.

\section{Melodic and Temporal Accent Structures}

Most compositions within Western music are generated from a diatonic scale that not only anchors a melody to a given tonality (e.g., C, in a melody based on the $\mathrm{C}$ major diatonic scale) but also constrains which notes can permissably appear, as well as their harmonic relationship to one another. Sequences of individual notes are then nested within melodic phrases of a longer time span. The endings of these phrases are marked by melodic accents that represent a change from the preceding context and thereby capture a listener's attention. In general, melodic accents are attributed to three types of structural changes (Benjamin, 1984; Berry, 1976; Cooper \& Meyer, 1960; Jones, 1987a).

The first are harmonic accents, in which members of the tonic triad chord are used to mark the ending of one melodic theme variation before shifting to another. In Figure 1A, for example, the sequence of notes is based on the $\mathrm{C}$ major scale and arranged into mensural groupings (demarcated by bar lines) that contain three notes each. Notice that the ending of every other measure is marked by a harmonic accent (h) that coincides with the tonic $(C)$, dominant $(\mathrm{G})$, mediant $(\mathrm{E})$, and tonic, respectively. In addition to outlining melodic theme organization, harmonic accents also reaffirm the melody's underlying tonality scheme and provide momentary points of resolution within the tune's total time span (Boltz, 1989; Kramer, 1982; Meyer, 1956).

A second means by which melodic phrase structure can arise is through pitch skips, which are denoted as (p) in Figure 1A. These types of accents represent a change in interval distance in which a significant deviation in pitch magnitude occurs. For example, notice that notes within the mensural groupings of Figure 1A are related by adjacent scale steps but notes between these groupings are separated by two to five scale steps.

The third and final type of melodic accent refers to changes in contour (c), or a shift from a rise in pitch to a fall, and vice versa. The melody in Figure 1 A, for example, initially ascends in pitch but then shifts in direction after the sixth note to descend in pitch, ascends, and then descends again to eventually resolve on the tonic interval.

These three types of melodic accents frequently coincide with one another, as is shown in Figure 1A. Pitch skips and contour changes, in particular, often occur immediately after harmonic accents and thereby accentuate the beginning of new theme variations. However, pitch skips and contour changes can also mark melodic phrase endings that are nested within higher order ones marked by harmonic accents. In addition, it is not necessarily true that all three types of accents are always present within a melody, and so, one accent type alone may be solely responsible for delineating melodic phrase boundaries. Despite their relative arrangement to one another, each type

\section{A}

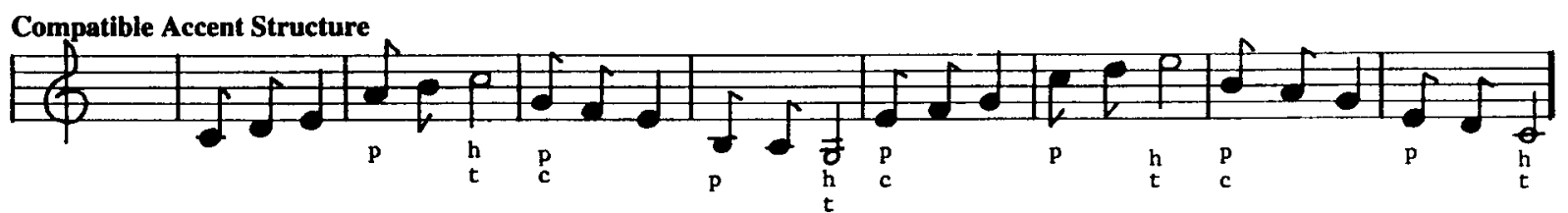

B

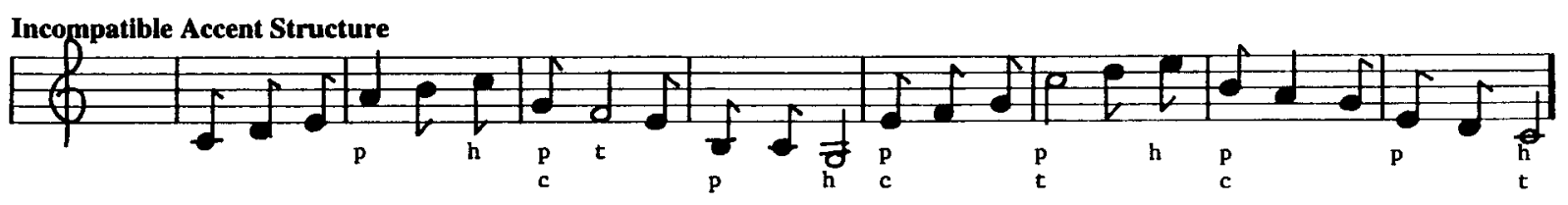

Figure 1. The melody shown in panel $A$ contains a compatible accent structure in that temporal accents, $t,(b)$ regularly recur and coincide with three types of melodic accents-namely, pitch skips (p) due to rule breaks, contour changes (c), and harmonic accents (h) marked by tonic triad members. In contrast, the melody in panel $B$ displays an incompatible accent structure because, even though temporal accents regularly recur, they fail to coincide with melodic accents. The result is that the melody's underlying organization of pitch relations is obscured. 
of melodic accent has been found to be perceptually salient to listeners and to guide selective attending and the generation of melodic expectancies (see Jones, 1987b, for a review). Perhaps more important, melodic phrase structure provides an overall organizational scheme that serves to link both adjacent and nonadjacent notes to a common framework. This, in turn, facilitates both learning and the remembering of melodic information (Boltz, 1991a; Boltz \& Jones, 1986).

Music, of course, is not only structured in space (i.e., pitch) but also in time. And across several different analyses of musical structure (see, e.g., Boltz \& Jones, 1986; Deutsch \& Feroe, 1981; Lerdahl \& Jackendoff, 1983; Yeston, 1976), it has been observed that a melody's temporal information bears a very lawful relationship to the organization of melodic information. Music displays a number of temporal dimensions, but the most common characterizations are in terms of meter, tempo, and rhythm. Meter provides the referent beat of a melody and, therefore, determines the base tempo in which the melody will unfold. For example, a meter based on a quarter note, $d$, as the referent beat will unfold at a relatively slower rate than one based on an eighth note, $\delta$. Against this background beat, rhythm introduces durational changes to a melody, because some notes receive more or fewer beats than the referent. From a psychological perspective, rhythm is important, because pauses and prolonged durations give rise to temporal accents that stand out from the melody's context and thereby capture a listener's attention. More important, temporal accents tend to coincide with melodic ones such that a joint accent structure results (Benjamin, 1984; Berry, 1976; Jones, 1987a; Lerdahl \& Jackendoff, 1983). In Figure 1A, for example, notice that temporal accents $(\mathrm{t})$ arising from the relatively longer half notes $(d)$ regularly recur after every six notes and coincide with melodic phrase boundaries that are marked by pitch skips, contour changes, and tonic triad members. This type of temporal accent structure serves to attentionally highlight a melody's organization of pitch relations and enhances the probability that listeners will become attuned to this organization during selective attending. However, in addition to rhythm, there is a second type of temporal change that typically reinforces the structural salience of melodic phrase structure. That is, most performers tend to display ritards, or decelerations of tempo, at melodic phrase endings and then a return to the base rate at the onset of a new phrase (Kronman \& Sundberg, 1987; Repp, 1992; N. Todd, 1985). The magnitudes of these ritards are directly proportional to the relative importance of melodic phrase endings: Decelerations are most pronounced at the very end of a piece and become increasingly less so as the hierarchical nesting of phrases decreases toward lower levels of a shorter time span.

From a behavioral perspective, joint accent structures facilitate cognitive performance in a number of different ways. Given that accents outline a melody's inherent structure, this, in turn, enhances the subsequent remembering of both melodic (Boltz, 1991a; Boltz \& Jones, 1986;
Deutsch, 1980) and temporal information (Boltz, 1992, 1995, 1998). In addition, others have suggested that joint accent structures facilitate attentional tracking and allow listeners to generate expectancies about both the what and the when of upcoming structural relations (Boltz \& Jones, 1986; Jones, 1981; Jones \& Boltz, 1989). This thereby enables listeners to coordinate their motor behavior with others in musical performance (Rasch, 1979, 1988) and enhances the ability to detect any deviant pitch intervals or tonal mistakes (Boltz, 1993; Jones, Boltz, \& Kidd, 1982).

\section{EXPERIMENT 1}

The primary question investigated in the present set of experiments is whether temporal/melodic accent structure also influences tempo discrimination. Although effects from accent compatibility are examined in Experiment 2, Experiment 1 is designed to examine the simpler case in which melodic accents alone are used to demarcate a melody's phrase structure against a background beat of an isochronous rhythm (i.e., no temporal accents). In addition, only two types of accents are manipulatednamely, contour changes that vary in their relative number and pitch skips that vary in their relative magnitude.

First, consider the case in which listeners are presented with a pair of melodies that are identical in their overall tempo and total duration but one tune contains more contour changes than the other. Is this melody judged to transpire more slowly than one with fewer shifts in pitch direction? If listeners have internalized those temporal changes that typically accompany melodic phrase endings, this type of finding may, in fact, occur. That is, even though rhythm and tempo are held constant throughout a melody's total time span, listeners may erroneously perceive prolonged accents and ritards at phrase ending points, and the more of these that are present, the slower the melody should seem. In addition, this effect should be enhanced or reduced when this melody is actually slower or faster, respectively, than the other.

A similar finding may occur when manipulating the magnitude of pitch skips that occur between successive tonal groups. Relative to small pitch skips that span one to two scale steps, those that span a greater interval distance should emerge as melodic accents marking phrase boundaries. If temporal changes are once again misperceived to accompany these phrase ending points, melodies displaying large pitch skips should be judged to be relatively slower than those with small pitch skips.

These two issues were investigated by presenting the subjects with a paired-comparison task in which the comparison melody of a pair differed from its standard in terms of the relative number of contour changes (same, more, fewer), magnitude of pitch skips (same, larger, smaller), and overall tempo (same, faster, slower). These manipulations demanded a rigorous experimental control of melodic structure, and, to this end, all the melodies within this study were generated from the next rule within 
A. Small Pitch Skips

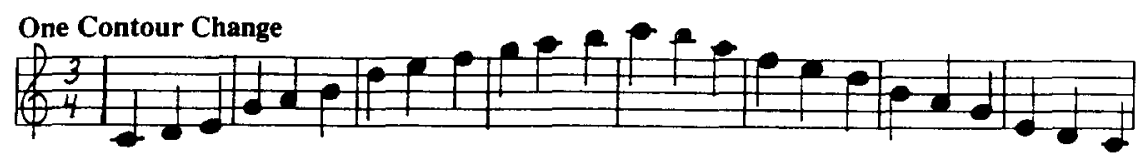

Three Contour Changes

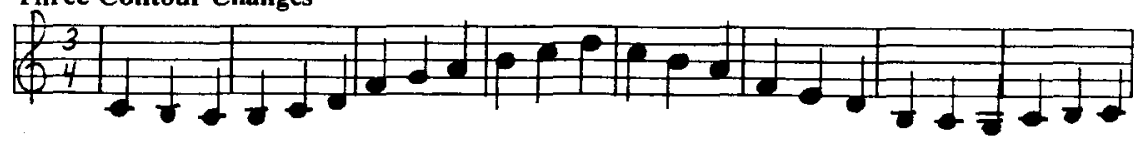

Five Contour Changes

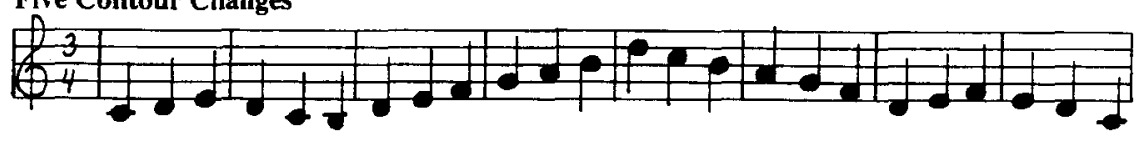

Seven Contour Changes

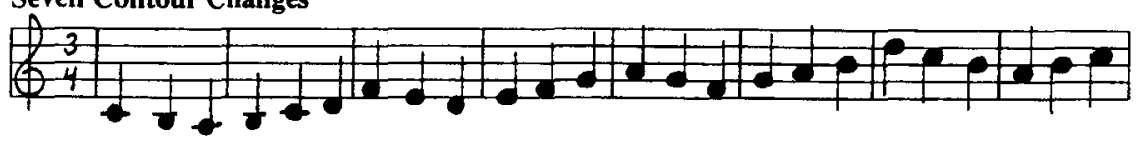

B. Large Pitch Skips
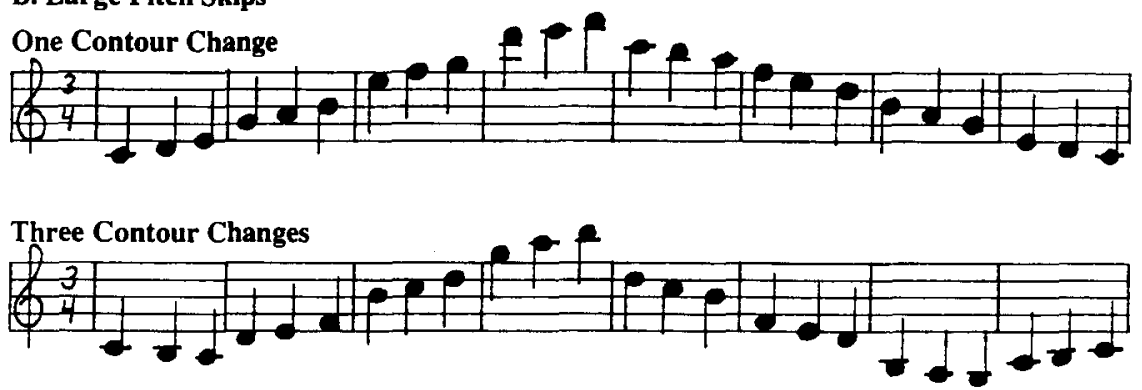

Five Contour Changes

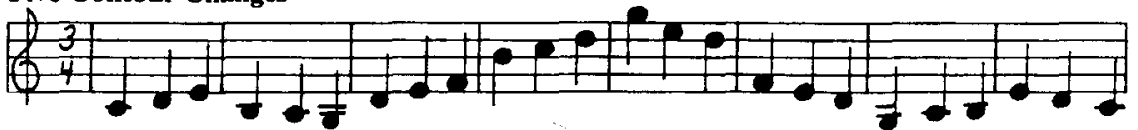

Seven Contour Changes

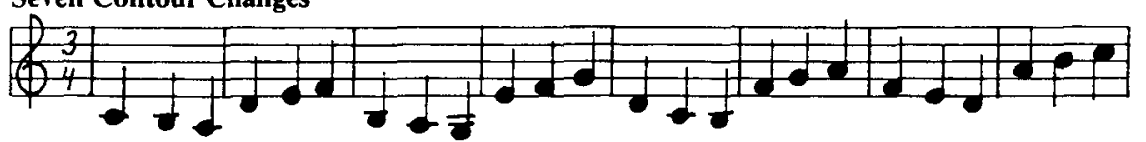

Figure 2. The set of musical patterns used in Experiment 1. All melodies are generated from the next rule within the dihedral symmetry group, $D_{7}$, and contain 24 notes arranged into eight sets of three-tone groups. Notes within a group (demarcated by bar lines) are related by adjacent scale steps $\left(\mathrm{N}^{ \pm 1}\right)$ but display a pitch skip between successive groups. Those melodies shown in panel $A$ contain a relatively small pitch skip $\left(N^{ \pm 1}\right.$ or $N^{ \pm 2}$ ), whereas those in panel $B$ display a relatively larger one (ranging between $N^{ \pm 2}$ and $N^{ \pm 5}$ ).

the dihedral symmetry group $\mathrm{D}_{7}$ (see Boltz \& Jones, 1986, for a more detailed discussion). As can be seen in Figure 2 , each melody contained 24 notes arranged into eight sets of three-tone groups (demarcated by bar lines) such that, within a given group, notes always represented adjacent scale steps that moved in a consistent direction. Each tonal group, however, was separated from its immediately preceding one by a pitch skip that led either to a continuation of the ongoing contour or to a shift in pitch direction. The subjects' task on each trial was to judge the tempo of the comparison melody, relative to the standard.

\section{Method}

Design and Subjects. The design was a $3 \times 7 \times 4 \times 2$ mixed factorial. Subjects received a series of paired melodies in which the standard and comparison could differ in tempo (same, faster, slower), number of contour changes $(0, \pm 2, \pm 4$, or \pm 6 changes $)$, and magnitude of pitch skips (small-same, large-same, larger, smaller). Counterbalance order (I, II) was the single between-subjects factor. 
Thirty-two subjects from an introductory psychology course at Haverford College participated in the experiment in return for course credit. All were nonmusicians with normal hearing abilities. Sixteen subjects were randomly assigned to one of the two counterbalance orders.

Stimulus materials. An initial set of eight musical patterns was constructed, each pattern containing 24 notes drawn from the $\mathrm{C}$ major diatonic scale. These patterns, which were generated from the next rule within the dihedral symmetry group $\mathrm{D}_{7}$, consisted of eight sets of three-tone groups. As can be seen in Figure 2, notes within a tonal group were related by the $\mathrm{N}^{ \pm 1}$ rule (representing adjacent scale steps), and notes between groups were separated by a pitch skip that varied in its magnitude. For the four melodies shown in the top panel of Figure 2, the pitch skip between groups was a relatively small one that could range between one or two scale steps (i.e., from $\mathrm{N}^{ \pm 1}$ to $\mathrm{N}^{ \pm 2}$ ). In contrast, the four melodies shown in the lower panel of Figure 2 displayed larger pitch skips that could vary between two and five scale steps (from $\mathrm{N}^{ \pm 2}$ to $\mathrm{N}^{ \pm 5}$ ). To establish a sense of tonality, each melody both began and ended on the tonic (C). However, within a melody, there were no harmonic accents (marked by tonic triad members) to provide momentary points of resolution.

As can be seen in Figure 2, the melodies also varied in terms of their number of contour changes. Within each pitch skip condition, the melodies contained one, three, five, or seven changes in pitch direction (i.e., a rise to a fall in pitch, or vice versa) whose overall shape was both symmetrical in nature and comparable to one another across the two conditions. These eight melodies were then transposed to the $G$ major diatonic scale to yield a total set of 16 melodic patterns.

For the paired comparison task, melodies were paired and arranged into two randomized orders (I, II) of 128 trials. Melodies within a pair were always based on the same key ( $\mathrm{C}$ or $\mathrm{G}$ major) but varied from one another in terms of their relative number of contour changes and magnitude of pitch skips. With respect to the latter, either the standard and comparison melodies both displayed large or small pitch skips (i.e., small-same; large-same) or the comparison contained larger or smaller pitch skips than did the standard (i.e., small-large; large-small). Within each counterbalance order, these were evenly distributed among the 128 trials, so that there were 32 trials within each of the four conditions. In addition, within each of the four pitch skip conditions, four melodic pairs were assigned to one of eight conditions of contour change. Relative to the standard, the comparison either contained the same number of contour changes (i.e., $1-1 ; 5-5$ ), two more or fewer changes (i.e., 3-5; 5-3), four more or fewer changes (i.e., 3-7; 7-3), or six more or fewer changes (i.e., 1-7; $7-1$ ). These particular pairings were selected in order to ensure that each melody appeared an equal number of times across the total set of experimental trials.

Lastly, the melodies within a given melodic pair always displayed an isochronous rhythm and one of two base rates. In one, the stimulus onset asynchrony (SOA) value for all notes was $375 \mathrm{msec}$ ( $250 \mathrm{msec}$ on-time and $125 \mathrm{msec}$ off-time), whereas, in the second, the SOA value was $525 \mathrm{msec}$ ( $350 \mathrm{msec}$ on-time and $175 \mathrm{msec}$ offtime). Within a pair, the comparison melody contained either the same base tempo as the standard or a tempo that was $12 \%$ faster or slower than the SOA of the base value. This transformation preserved the original ratio of on- to off-times and is based on the past literature (Drake \& Botte, 1993), which suggests that a $12 \%$ difference should be sufficient for subjects to detect a change. For each counterbalance order, $50 \%$ of the melodic pairs always displayed the same tempo, whereas $25 \%$ of the trials displayed a faster or slower tempo difference, respectively. This relative distribution was also reflected in the eight contour change conditions that were nested within each of the four pitch skip conditions.

Apparatus. All melodies were constructed and generated with the Midilab software system (R. E. Todd, Boltz, \& Jones, 1989). Dur- ing each experimental session, melodies were presented on line by a Yamaha TX81Z FM tone generator controlled by an IBM AT computer with a Roland MPU-401 MIDI interface unit. Sequences of tones were amplified by a Kenwood KR-A4010 receiver and played over Koss Pro 4AAA Plus headphones at a comfortable listening level $(60 \mathrm{~dB})$. The timbre of all melodies was that of a grand piano.

Procedure. The subjects were tested over a 2-day period. On the 1 st day, recorded instructions informed the subjects of pattern presentation details and task requirements. On each trial, a l-sec warning tone $\left(\mathrm{C}_{7}-\mathrm{C}\right.$ in the seventh octave $)$ preceded the standard melody of a pair by $2 \mathrm{sec}$. Three seconds after the offset of this standard sequence, a comparison melody was played. During the 5 -sec response period that followed, the subjects were asked to judge the apparent tempo of the comparison melody, relative to that of the standard, on a 7-point rating scale. They were told that, if the comparison seemed much faster (or slower), they should select a value of -3 (or +3 ), and if the comparison seemed only somewhat faster (or slower), a value of -2 or -1 (or +2 or +1 ) would be appropriate. A value of zero in the middle of the scale indicated that the two melodies seemed equivalent in their overall tempo. The subjects made these judgments on a response console, which were then automatically recorded by the computer. The procedure for Day 2 was identical to that for Day 1, except that, instead of receiving the full set of instructions, the subjects were merely reminded of their rating task.

All the subjects were tested in small groups of 2-4 individuals. On each day, they received 6 practice trials, followed by the set of 128 experimental trials, with a brief rest break provided after the first 64 trials. Those subjects receiving Order 1 on Day 1 received Order 2 on Day 2, and vice versa. Each experimental session was approximately $90 \mathrm{~min}$ in duration.

\section{Results}

An initial analysis of variance (ANOVA) was conducted on the subjects' ratings and revealed no significant effects of either counterbalance order or base tempi. Given this, all subsequent analyses were collapsed over these two variables. In addition, for purposes of simplicity, the first analysis was collapsed over the different degrees of contour change between standard and comparison melodies (i.e., $\pm 2, \pm 4, \pm 6$ ) such that the resulting statistical design was a repeated measures factorial with three independent variables: tempo (same, slower, and faster in comparisons), contour differences (same, more, and fewer changes in comparisons), and magnitude of pitch skips (small-small, large-large, small-large, largesmall) between standard and comparison melodies. The mean tempo ratings for these different conditions are depicted in Figure 3.

The overall ANOVA revealed that the most important findings were the effects of a melody's pitch properties on perceived rate. A main effect for contour difference $\left[F(3,180)=11.03, M S_{\mathrm{e}}=0.46, p<.001\right]$ indicated that, across all conditions of the experiment, comparison melodies containing more contour changes than did the standard were judged to be significantly slower than pairings containing an equivalent number of contour changes. Conversely, comparisons containing fewer changes than did the standard were judged to be significantly faster. These differences were confirmed by a set of Tukey post hoc comparisons when $p$ was set at .05 . In addition to contour, tempo ratings also varied because of a sig- 
A. Comparison Melody Same Tempo as Standard

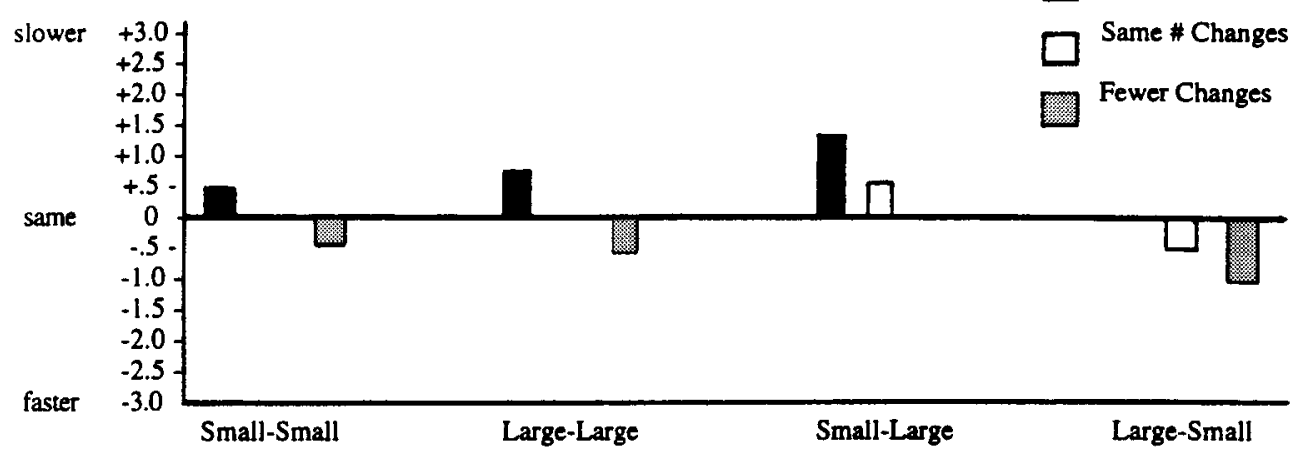

Magnitude of Pitch Skips Between Standard and Comparison Melodies

B. Comparison Melody Slower Than Standard

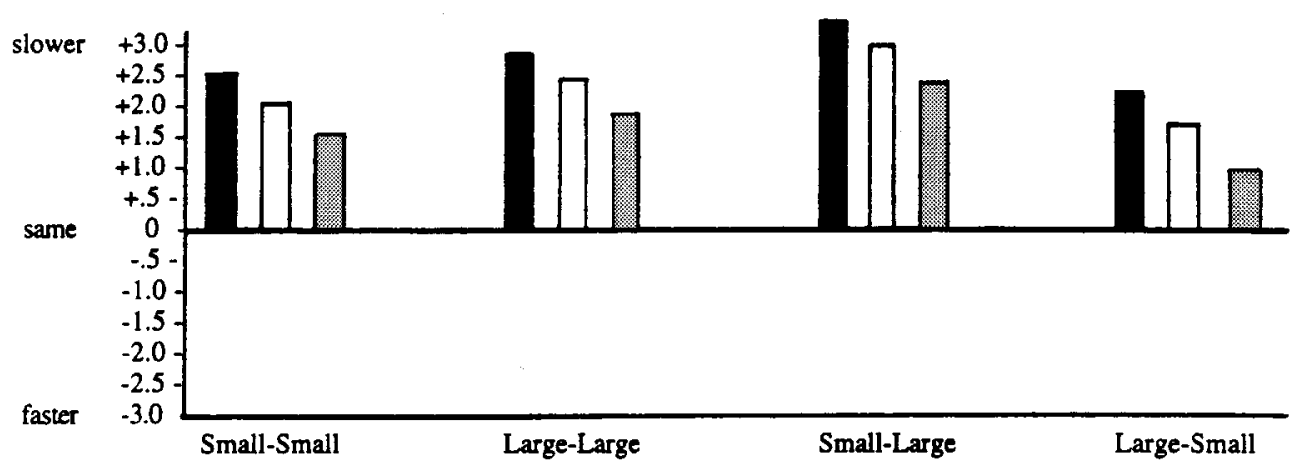

Magnitude of Pitch Skips Between Standard and Comparison Melodies

\section{Comparison Melody Faster Than Standard}

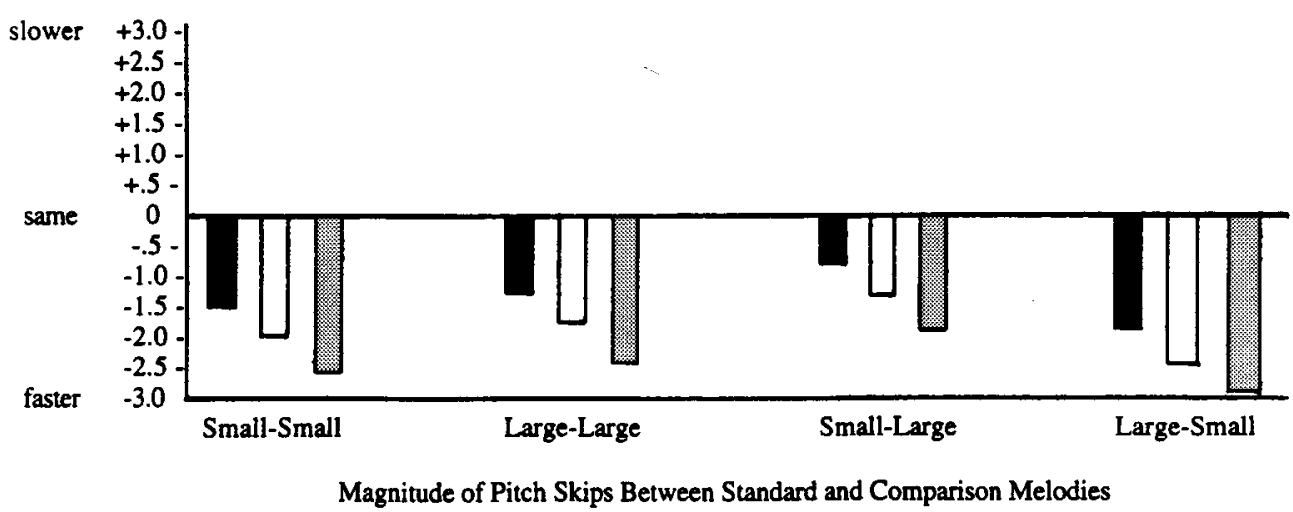

Figure 3. Mean tempo ratings in Experiment 1, as a function of variations in tempo, relative number of contour changes, and the magnitude of pitch skips in comparison as opposed to standard melodies.

nificant main effect of pitch skips $\left[F(3,180)=5.6, M S_{\mathrm{e}}=\right.$ $0.51, p<.001]$. A set of Tukey post hoc comparisons $(.05)$ indicated that, relative to melodic pairs containing comparable spans of pitch distance (small-small, large-large), those in which the comparison contained larger pitch skips than did the standard were judged to be significantly slower, whereas those containing smaller pitch skips were judged to be significantly faster. Lastly, a significant interaction between contour differences and pitch skips $\left[F(9,540)=9.76, M S_{\mathrm{e}}=0.47, p<.001\right]$, revealed that these two variables exerted a joint impact upon one another. This effect is one that generalized to each of the three tempo conditions in Figure 3. As can be seen, relative to melodic pairs containing the same number of con- 
tour changes and a comparable magnitude of pitch skips, those containing more contour changes or larger pitch skips alone in comparison than in standard melodies led to significantly slower tempo ratings $(p<.05)$. In addition, these effects were significantly enhanced $(p<.05)$ when the comparison displayed both of these properties together. The opposite effect occurred when the temporal ordering of these melodic pairings was reversed. That is, comparison melodies containing both fewer contour changes and smaller pitch skips than did the standard were rated significantly faster than comparisons containing one of these attributes alone $(p<.05)$. These, in turn, were rated as being significantly faster than melodic pairings containing the same number of contour changes and a comparable magnitude of pitch skips $(p<.05)$. These effects, however, could be reduced by the presence of the other variable. For example, comparisons containing more contour changes but smaller pitch skips than did the standard were rated identically to melodic pairs containing the same number of contour changes and equivalent spans of pitch skips. The same was true when comparisons contained fewer contour changes but larger pitch skips than did the standard. In effect, then, the influence of one variable cancelled out the influence of the other.

These effects of pitch on tempo were, once again, relatively robust ones that generalized across the manipulation of tempo. Although the absolute value of ratings varied across the three panels of Figure 3, this was due to a significant main effect of tempo $[F(2,90)=52.1$, $\left.M S_{\mathrm{e}}=0.67, p<.001\right]$. Relative to the same tempo condition, comparison melodies that were actually faster or slower than the standard were, in fact, perceived as such by the subjects. The joint effects of contour change and pitch distance, however, are particularly noteworthy in the same tempo condition in which there were no actual

Table 1

Percent Frequency of Same, Slower, and Faster Responses Within the Same Tempo Condition of Experiment 1, as a Function of Variations in the Relative Number of Contour Changes and Magnitude of Pitch Skips Between Comparison and Standard Melodies

\begin{tabular}{lrrr}
\hline & \multicolumn{3}{c}{ Subjects' Responses } \\
\cline { 2 - 4 } \multicolumn{1}{c}{ Pitch Skips } & Same & Slower & Faster \\
\hline Small-Small & 35 & 63 & 2 \\
$\quad$ More contour changes & 98 & 1 & 1 \\
$\quad$ Same number of contour changes & 39 & 3 & 58 \\
$\quad$ Fewer contour changes & & & \\
Large-Large & 40 & 55 & 5 \\
$\quad$ More contour changes & 99 & 1 & 0 \\
Same number of contour changes & 37 & 3 & 60 \\
Fewer contour changes & & & \\
Small-Large & 26 & 74 & 0 \\
$\quad$ More contour changes & 42 & 56 & 2 \\
Same number of contour changes & 66 & 10 & 24 \\
Fewer contour changes & & & \\
Large-Small & 58 & 27 & 15 \\
$\quad$ More contour changes & 45 & 5 & 50 \\
$\quad$ Same number of contour changes & 27 & 2 & 71 \\
Fewer contour changes & &
\end{tabular}

tempo differences between standard and comparison melodies. This illustrates, then, that an apparent change in the velocity of auditory motion can be attributed to pitch properties alone. To determine the extent to which this effect generalized to all the subjects in the experiment, the percent frequency of same, slower, and faster responses was determined for each pitch condition of those melodic pairings containing equivalent tempi. These are shown in Table 1.

The results, in general, reveal that the interaction between contour change and pitch distance applied to most subjects. In the two conditions in which the standard and comparison melodies displayed a comparable magnitude of pitch skips (small-small, large-large), the subjects correctly judged melodies to be equivalent in tempo when both contained the same number of contour changes. However, relative to these conditions, the subjects assigned a significantly higher percentage of slower responses to comparison melodies containing more contour changes than did their standards and a higher percentage of faster responses to comparisons containing a relatively fewer number of contour changes.

In the lower half of the table, it is apparent that the subjects' responses were also influenced by differences in pitch skips within a given melodic pair. Here, the number of correct same responses markedly declines, because the subjects assigned slower tempi to comparison melodies containing larger pitch skips, particularly when these were accompanied by more contour changes. The reverse occurred when comparisons displayed smaller pitch skips, which produced a high frequency of faster responses, particularly when these also displayed relatively fewer contour changes.

The more interesting finding occurred when melodies contained more contour changes but smaller pitch changes, and vice versa. In each case, the percentage of same responses increased, but notice that, of the two variables, contour change appeared to exert a greater impact on judgments than did the magnitude of pitch skips. For example, in the small-large condition with fewer contour changes, those subjects who did not assign same ratings could either judge a comparison melody to be slower because of large pitch skips or faster because of fewer contour changes. As seen in the table, most subjects opted for the latter. A similar finding is observed in the large-small condition with more contour changes. Here, more subjects selected the slower response category associated with more contour changes than the faster category associated with smaller pitch skips.

A final analysis was conducted on these data; it was designed to determine whether the subjects' tempo ratings not only varied with tempo and the magnitude of pitch skips but also with the magnitude of contour change between standard and comparison melodies. The overall analysis revealed that this was the case. A main effect for contour change $\left[F(7,180)=9.35, M S_{\mathrm{e}}=0.23, p<.001\right]$ indicated that, in general, a difference of at least four 
contour changes between standard and comparison melodies was required to significantly influence tempo judgments. In addition, a set of Tukey post hoc comparisons $(p<.05)$ indicated that, although tempo ratings between the 0 and \pm 4 and the \pm 2 and \pm 6 change conditions were significantly different from one another, those between the 0 and \pm 2 and the \pm 4 and \pm 6 change conditions were not. This pattern of differences also applied to the significant interaction between contour change and magnitude of pitch skips $\left[F(21,540)=10.41, M S_{\mathrm{e}}=.18, p<\right.$ $.001]$. Although successive increases (or decreases) in the number of contour changes consistently led to increasingly slower (or faster) tempo ratings, a difference of four was once again required for significance.

\section{Discussion}

The primary finding from this first experiment is that a melody's arrangement of pitch relations can influence its apparent tempo. Overall, those patterns containing more changes in pitch direction and a greater magnitude of pitch skips were judged to unfold more slowly than those displaying a comparable number of contour changes and equivalent spans of pitch distance. Conversely, melodies containing a relatively fewer number of contour changes and smaller pitch skips than the immediately preceding referent were judged to unfold more quickly. Unlike the results of some paired-comparison tasks (Hellstrom, 1985), no temporal order effects were observed. Results were symmetrical in that increases in contour and pitch change produced the same magnitude of effect on tempo ratings as did successive decreases. Lastly, the influence of these two variables were both additive and subtractive in nature. The presence of contour and pitch change together significantly enhanced ratings, relative to the presence of one factor alone, and, in the case of conflicting information (i.e., more contour changes but smaller pitch skips, or vice versa), one factor served to cancel out the effects of the other, yielding ratings comparable with those for melodic pairings containing similar arrays of pitch information.

These phenomena were relatively robust ones that generalized across several different contexts. They applied to different melodic keys ( $\mathrm{C}$ and $\mathrm{G}$ major), to different base tempi (SOAs of 375 and $525 \mathrm{msec}$ ), to a majority of subjects in the experiment, and to melodic pairings showing both null and actual tempo differences. This latter finding is a relatively important one, because it indicates that pitch was able to induce perceptual tempo differences when none existed, even when the remaining $50 \%$ of the experimental trials displayed actual tempo differences. This, in turn, suggests that effects were not simply due to response bias but are reliable phenomena in the perception of auditory motion.

From a theoretical perspective, these overall results suggest that people's past experience with certain structural invariants within Western music can give rise to illusory tempo changes. Within most musical compositions, melodic phrase endings marked by members of the tonic triad chord, significant pitch skips, or changes in pitch direction are typically accompanied by prolonged tonal durations and tempo decelerations. This type of structural relationship is assumed to be particularly adaptive for cognitive performance, by drawing listeners' attending to the inherent organization of a melody and the interrelationships among tonal groupings. The previous literature indicates that this is, in fact, the case, in that joint accent structures not only facilitate learning and remembering (Boltz, 1992, 1995, 1998; Deutsch, 1980) but provide structural referents for melody recall (Boltz, 1991 a; Boltz \& Jones, 1986) and musical performance (Schmuckler, 1990) as well as selective attending (Boltz, 1993). The present results extend this research by suggesting that people sometimes overgeneralize the joint relationship between temporal and melodic accents by perceiving the expected temporal changes at melodic accent points, even when these are physically absent. Although all melodies here displayed a uniform tempo and rhythm throughout a tune's total time span, those compositions containing more contour changes or larger pitch skips than did their respective standards were judged to be significantly slower. In addition, these effects were enhanced when contour and interval accents together rendered melodic phrase structure more salient but reduced when melodic phrasing was more ambiguous (e.g., more contour changes but smaller pitch skips). The third type of melodic accent, arising from tonic triad members, was not manipulated but, nonetheless, should yield a similar pattern of results. In fact, given that harmonic accents are judged to provide momentary points of tonal resolution, their effects on tempo perception may be even more pronounced than those stemming from contour and interval accents. In sum, then, the approach being offered here suggests that, through repeated exposure to Western music, people eventually become attuned to a composer's use of joint accent structures in outlining the inherent organization of a melody's relationships. Although this can benefit cognitive behavior in several different ways, one consequence is that people may perceive tempo accelerations or decelerations even when no such changes are physically present.

One finding that merits some discussion is that contour change exerted a greater effect on subjects' tempo ratings than did the magnitude of pitch skips. There are two alternative interpretations of this result. One is that directional changes may simply have a greater impact than does pitch distance on the perception of auditory motion. A more likely explanation, however, is methodological in nature, in which the difference in the number of contour changes between standard and comparison melodies may have been more salient to listeners than the difference in pitch change. The former could span over a relatively wide range of values $(0, \pm 2, \pm 4, \pm 6)$ and, in fact, a difference of at least four contour changes was required in order to influence tempo ratings. The manipulation of pitch change, on the other hand, was much more limited, in that there were only two levels of this variable (small and large) and the magnitude of difference be- 
tween these levels was not a particularly pronounced one (i.e., $\mathrm{N}^{ \pm 1}$ to $\mathrm{N}^{ \pm 2}$ for the former vs. $\mathrm{N}^{ \pm 2}$ to $\mathrm{N}^{ \pm 5}$ for the latter). If one were to replicate this study using multiple levels of pitch change and ones that spanned over a greater range of pitch distance, it is quite likely that more robust effects would emerge.

\section{EXPERIMENT 2}

The purpose of Experiment 2 is to investigate whether the temporal accentuation of certain pitch relations can influence the perceived rate at which a melody unfolds. Although several studies have investigated the effects of tempo on rhythm perception or performance (Clarke, 1982; Handel, 1993; Marshburn \& Jones, 1985; Povel, 1977), very few have examined the reverse relationship of how rhythm may influence tempo. One is an experiment by Wang (1984), who asked subjects to listen to two different rhythmic versions of Dvorak's Humoreske. The first represented the original composition of the piece, in which the underlying rhythm was termed uneven because it displayed multiple changes in duration (i.e., whereas the second was termed even because it displayed an isochronous rhythm (i.e., ...... Te Tempo changes were introduced after a varying number of notes in the piece, and the subjects' task was to detect these as quickly as possible. Relative to the even rhythm, the results showed that it took more time to discriminate tempo changes in the uneven rhythm. A second study, in which the effects of rhythm on tempo perception were examined, was conducted by Drake and Botte (1993). Here, the pitch properties of melodies were always held constant, but patterns could contain either a regular or an irregular rhythm. In the former, the interonset interval (IOI) between tones was a fairly lawful one that either alternated between longshort or short-long values or became increasingly longer or shorter as the tune unfolded. In contrast, the IOI values in irregular rhythms displayed no lawful patternings. The subjects were presented with pairs of patterns that varied in their actual tempi, and the results showed that the JNDs of these tempo discriminations were much lower for patterns containing regular rhythms.

In sum, then, these two studies together suggest that patterns of durational change within a melody can influence both the accuracy and the reaction time in which tempo changes are detected. One issue, however, that remains unclear is whether a melody's rhythm relative to the arrangement of pitch relations can influence the rate at which the melody is perceived to unfold. In the Drake and Botte (1993) study, the primary intent was to assess the effects of temporal patterning alone on discrimination, and so, pitch relations were therefore held constant (i.e., monotonic). Similarly, in the Wang (1984) study, the relationship of rhythm to the melodic line was never manipulated, because the uneven rhythm represented the original composition (whose relation to pitch information was never described) and the even rhythm was simply an isochronous sequence with no temporal accentuation. Hence, it is not known whether the attentional highlighting of certain pitch intervals can influence behavior in such a way that two melodies with the same tempo but different rhythms may be perceived to have different rates or whether the overall speed of a given melody can be enhanced or reduced by rhythm. Given the notion of joint accent structure and the results of Experiment 1, there is reason to believe that this may be the case.

In many, if not most, musical compositions, temporal accents in the form of prolonged durations do coincide with melodic accents marking phrase boundaries, as was illustrated earlier in Figure 1A. However, this is not always the case. In some instances, the pattern of accented and unaccented notes (i.e., rhythm) may be incompatible with the arrangement of melodic structure. This is particularly apparent at early stages of learning. For example, the music of a novice pianist will often display a pattern of temporal accents that fails to coincide with melodic ones. Such a melody is represented in Figure 1B. Notice that, although temporal accents are lawfully arranged, these conflict with contour change points, pitch skips, and those tonic triad members marking the ending of each tonal grouping. Given that melodic information and temporal information are structurally dissociated from one another and appear to lack a unified organizational scheme, one would expect this type of melody to disrupt cognitiveprocessing activities. The empirical evidence, in fact, shows that, relative to joint accent structures, those displaying conflicting accents not only impair learning and the subsequent remembering of melodic information but also attentional tracking and the perceptual pickup of information as well (Boltz, 1991a, 1992, 1993, 1995, 1998; Boltz \& Jones, 1986; Deutsch, 1980).

In addition to these effects on cognitive behavior, it may also be the case that compatible and incompatible rhythms will differentially influence tempo perception. Compatible accent structures yield temporal and melodic phrase groupings that mutually define and reinforce one another and that, from the perspective of a listener, should appear to smoothly unfold in a highly coordinated fashion. In contrast, melodies with incompatible accent structures contain temporal and melodic phrase groupings that are structurally dissociated from one another. In addition to unfolding in a seemingly disjointed fashion, these types of melodies display a greater number of phrase groupings, some of which are marked by melodic accents alone and others by temporal accents alone. This, in turn, may serve to decelerate judged tempo. If, as in Experiment 1 , listeners perceptually impose temporal accents on melodic accent points, melodies with incompatible rhythms should be judged to be significantly slower than those with compatible rhythms.

In Experiment 2, this issue was investigated by once again relying on a paired-comparison task. Unlike the previous experiment, melodies within a pair contained a comparable number of contour changes and range of pitch 
A. Melodies Based on 3-Tone Groupings

Tyo Contour Changes - Compatible Rhythm

年1

Two Contour Changes - Inverted - Incompatible Rhythm

Three Contour Changes - Compatible Rhythm

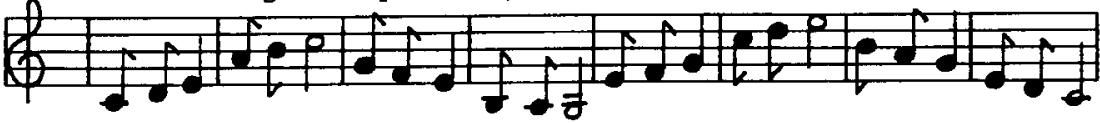

Three Contour Changes - Inverted - Incompatible Rhythm

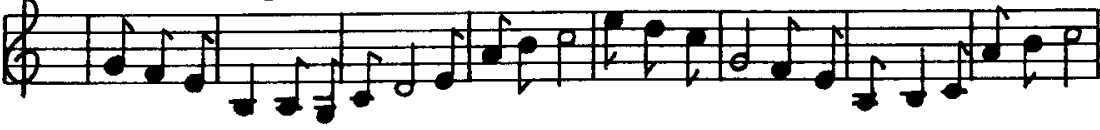

Five Contour Changes - Compatible Rhythm

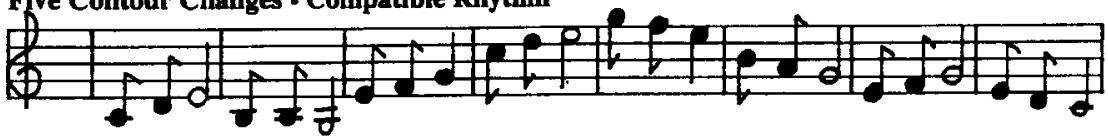

Five Contour Changes - Inverted - Incompatible Rhythm

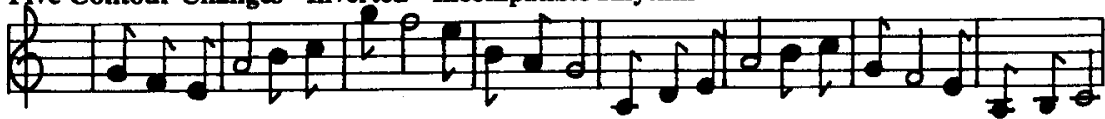

B. Melodies Based on 4-Tone Groupings

Two Contour Changes - Compatible Rhythm

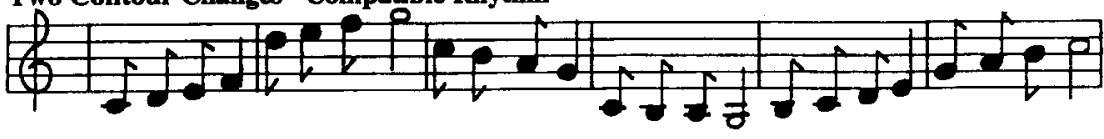

Two Contour Changes - Inverted - Incompatible Rhythm

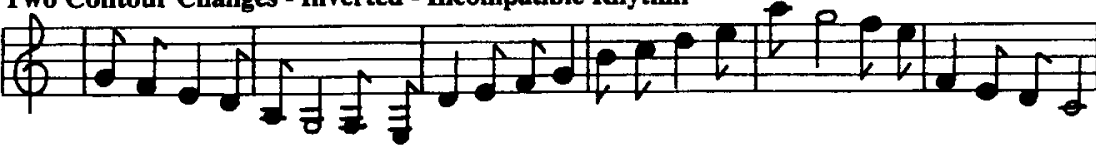

Three Contour Changes - Compatible Rhythm

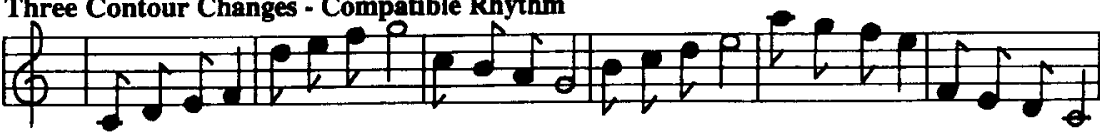

Three Contour Changes - Inverted - Incompatible Rhythm

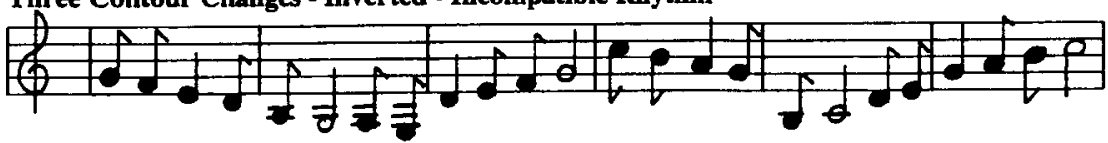

Five Contour Changes - Compatible Rhythm

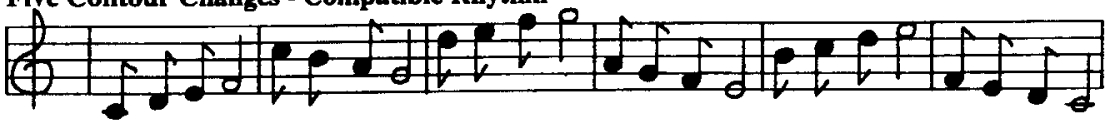

Five Contour Changes - Inverted - Incompatible Rhythm

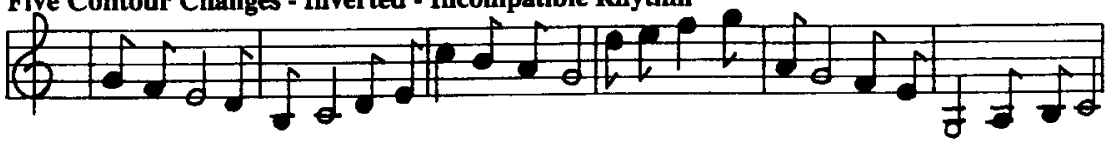

Figure 4. The set of musical patterns used in Experiment 2. 
skips. However, the pattern of temporal accentuation was varied, relative to the array of melodic accents, such that melodic pairs displayed one of four types of rhythmic relationships: compatible-compatible, incompatibleincompatible, compatible-incompatible, or incompatiblecompatible. The subjects' task was to rate the tempo of the comparison pattern, relative to that of the standard.

\section{Method}

Design and Subjects. The design was a $3 \times 4 \times 2$ mixed factorial. The subjects listened to a series of paired melodies in which the standard and comparison could differ in tempo (same, faster, slower) and the compatibility of rhythmic accent structure relative to melodic structure (compatible-compatible, incompatibleincompatible, compatible-incompatible, incompatible-compatible). The single between-subjects factor was counterbalance order (I, II).

Thirty-two subjects participated in the experiment and satisfied the same set of criteria as that in Experiment 1.

Stimulus materials. A set of 12 musical patterns was generated from the next rule within the dihedral symmetry group, $\mathrm{D}_{7}$, each pattern consisting of 24 notes drawn from the $\mathrm{C}$ major diatonic scale. As can be seen in the upper panel of Figure 4, half of the melodies were based on an initial three-tone argument $\left(\mathrm{C}_{4} \mathrm{D}_{4} \mathrm{E}_{4}\right.$, where subscripts indicate octave) and resulted in eight tonal groupings. The remaining half, shown in the lower panel of Figure 4, were based on an initial four-tone argument of $\mathrm{C}_{4} \mathrm{D}_{4} \mathrm{E}_{4} \mathrm{~F}_{4}$ and thereby resulted in six tonal groups. Notes within a group were always related by the $\mathrm{N}^{ \pm 1}$ rule representing adjacent scale steps, whereas the pitch skip between adjacent groups was of a greater distance that ranged between $\mathrm{N}^{ \pm 2}$ and $\mathrm{N}^{ \pm 6}$. In contrast to Experiment 1, the magnitude of these pitch skips was roughly comparable across all the melodies. In addition, not only did each melody resolve on the tonic interval (C), but the final note of each tonal group was marked by a tonic triad member (namely, the tonic, C; the mediant, E; or the dominant, $\mathrm{G})$ to provide momentary points of resolution as well as a reaffirmation of the melody's underlying tonality scheme.

In Figure 4, notice that the six melodies containing three- or fourtone groupings varied in their total number of contour changes. $\mathrm{Al}$ though the overall contour was always symmetrical in shape, two melodic instances contained either two, three, or five contour changes where one of the two instances was inverted relative to the other. For example, in the two contour change condition, one melody ascends, descends, and ascends again, whereas the second descends, ascends, and then descends toward the tonic ending. In addition, the melody containing the inverted contour shape always began on the dominant interval, $G_{4}$, and then moved downward by adjacent scale steps.

For each of the 12 melodies, two different rhythms were constructed. One was compatible with the arrangement of pitch information, in that temporal accents lawfully recurred and always coincided with pitch skips (i.e., rule breaks) and contour change points. Across all melodies, the referent background beat was an eighth note (.), and temporal accents were introduced by marking rule breaks that resulted in no contour change with a quarter note $(d)$ and those rule breaks accompanied by a contour change with a half note $(d)$. Rule breaks with contour changes, therefore, received greater accentuation than did rule breaks with no contour changes. The second type of rhythm was incompatible with the arrangement of pitch information, in that temporal accents did not consistently coincide with either pitch skips (rule breaks) or contour change points. Here, the relative frequency of eighth, quarter, and half notes was always the same as that in the corresponding compatible rhythm, and these too were arranged into a lawfully recurrent pattern. However, temporal accents $\left(\delta_{0}\right.$ or $)$ coincided with notes within a given tonal grouping, to obscure the underlying organization of pitch relations. The one exception was the presence of a temporal accent at the midpoint of a melody (i.e., at note 12), which was necessary in order to create a lawful rhythm.

The compatible and incompatible rhythms for each of the 12 melodies are shown in Figure 4. Although the incompatible rhythm is always assigned to the inverted melody in the figure, the two types of rhythms were actually imposed on all 12 melodies, yielding a total set of 24 patterns. It should also be mentioned that, in most cases, the set of rhythms did not result in a consistent meter for a given melody.

The particular durations assigned to a sequence of notes were generated from one of two base rates. One was relatively slower than the other in that the following tonal durations (on-times) and intertone intervals (off-times) were assigned to each type of note: eighth notes, $150 \mathrm{msec}$ on and $75 \mathrm{msec}$ off; quarter notes, $300 \mathrm{msec}$ on and $150 \mathrm{msec}$ off; and half notes, $600 \mathrm{msec}$ on and $300 \mathrm{msec}$ off. Notes in the second base rate corresponded to the following values: eighth notes, $120 \mathrm{msec}$ on and $60 \mathrm{msec}$ off; quarter notes, $240 \mathrm{msec}$ on and $120 \mathrm{msec}$ off; and half notes, $480 \mathrm{msec}$ on and $240 \mathrm{msec}$ off.

For the paired-comparison task, melodies were paired and arranged into two randomized orders (I, II) of 96 trials. Within a pair, melodies always shared the same number of contour changes and overall shape, but the contour of the comparison was the inverse of that of the standard. Melodic pairs varied from one another in terms of their rhythmic patterning. In particular, 24 of the 96 melodies in each order displayed one of four types of rhythm pairs for standard and comparison melodies, respectively: compatible-compatible, incompatible-incompatible, compatible-incompatible, or incompatible-compatible. Of the 24 melodies in each rhythm condition, 8 were assigned to each of the three contour change conditions (i.e., two, three, or five contour changes) such that 4 were based on melodies containing three-tone groupings and 4 were based on melodies with four-tone groupings. Lastly, half of the melodic pairs in a given counterbalance order displayed the same tempo, whereas $25 \%$ of the pairs contained a comparison melody that was either $12 \%$ faster or $12 \%$ slower than its standard. These tempo changes always preserved the underlying rhythm of a melody, and the relative distribution of melodic pairs displaying same, faster, or slower tempi was also reflected in the different contour change conditions nested within each of the four rhythm conditions.

Apparatus and Procedure. These were identical to those of Experiment 1.

\section{Results}

The subjects' mean tempo ratings as a function of variations in rhythmic accent structure and actual tempo differences between standard and comparison melodies are shown in Figure 5. Means have been collapsed over the two base tempi, counterbalance orders, and the number of contour changes across melodic pairs, since none of these variables exerted significant effects upon behavior $(F<1)$.

Results from the overall ANOVA indicated that the primary finding was a main effect of rhythmic structure $\left[F(3,180)=8.45, M S_{\mathrm{e}}=0.39, p<.001\right]$. Relative to those melodic pairs displaying a similar rhythm (compatiblecompatible, incompatible-incompatible), a set of Tukey post hoc comparisons revealed that comparison melodies displaying compatible accent structures were judged to be significantly faster than standard melodies containing incompatible accent structures $(p<.05)$. In addition, the opposite effect was observed, in which comparison melo- 


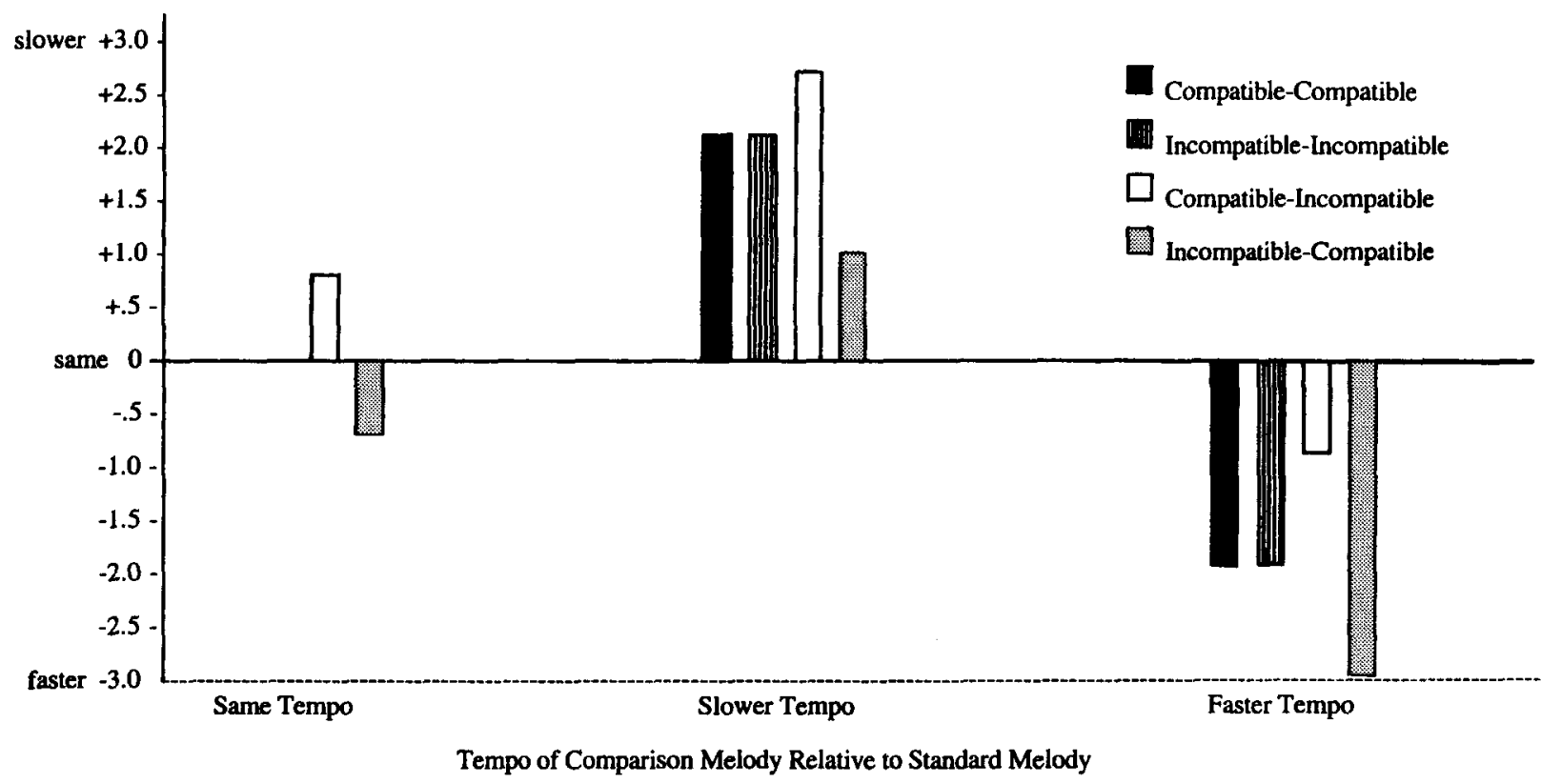

Figure 5. Mean tempo ratings in Experiment 2, as a function of variations in tempo and the rhythmic accent structure of standard as opposed to comparison melodies.

dies with incompatible rhythms were judged to be significantly slower than standard melodies with compatible rhythms $(p<.05)$.

Although this pattern of differences generalized across the three tempo conditions shown in Figure 5, the mean ratings in these latter conditions varied from one another, because of a main effect of tempo $\left[F_{(2,90)}=37.01, M S_{\mathrm{e}}=\right.$ $0.47, p<.001]$. Relative to the same tempo condition, those comparison melodies that were actually slower or faster than their standard melodies were, in fact, perceived as such by subjects $(p<.01)$.

In order to assess the effects of rhythm when null tempo differences actually existed, the percent frequency of same, slower, and faster responses was determined for the same tempo condition. As can be seen in Table 2, melodies were correctly judged to be equivalent in tempo when both contained the same rhythm. However, accuracy declined from $99 \%$ to $68 \%$ when melodies within a pair differed in their rhythmic accent structure. When errors occurred, the subjects were more likely to judge an incompatible rhythm to be slower than a compatible one and to be faster when the temporal ordering of rhythms was reversed.

\section{Discussion}

In sum, the present set of results illustrates that rhythmic accent structure can influence the apparent tempo of a melody. Rhythmic compatibility not only facilitates selective attending, learning, and remembering, as the past literature has indicated, but also accelerates the perceived tempo of musical patterns. From a psychological perspective, these different types of cognitive behaviors are all rendered by the structural consequences of joint accentuation. Patterns of temporal accentuation that directly coincide with melodic ones serve to highlight the number and location of phrase boundaries. This, in turn, directs attending toward different levels of a melody's organization and the interrelationships among both adjacent and nonadjacent intervals. In addition, given the lawful recurrence of temporal accents, listeners are able to extrapolate the preceding context and generate expectancies not only about when future accents will occur but also about what type of melodic information they will mark. Hence, as the melody unfolds over its total duration, it appears as a highly integrated event that smoothly flows along its predicted time course.

Incompatible accent structures, on the other hand, display temporal accents that may regularly recur, as in the present set of melodies, but nonetheless conflict with different levels of melodic structure. Given that the underlying organization of melodic relations is obscured and does not allow listeners to engage in anticipatory attending, these types of melodies will appear to consist of seem-

Table 2

Percent Frequency of Same, Slower, and Faster Responses Within the Same Tempo Condition of Experiment 2, as a Function of the Rhythmic Accent Structure Between Standard and Comparison Melodies

\begin{tabular}{lccc} 
& \multicolumn{3}{c}{ Subjects' Responses } \\
\cline { 2 - 4 } \multicolumn{1}{c}{ Rhythmic Pairs } & Same & Slower & Faster \\
\hline Compatible-compatible & 100 & 0 & 0 \\
Incompatible-incompatible & 98 & 2 & 0 \\
Compatible-incompatible & 67 & 33 & 0 \\
Incompatible-compatible & 69 & 2 & 29 \\
\hline
\end{tabular}


ingly disjointed and unrelated items. They will also be perceived to unfold more slowly than do melodies with compatible accent structures. In addition to actual prolonged durations arising from temporal accents, people appear to impose such changes on melodic phrase boundaries that are redundantly marked by pitch skips and contour changes as well as tonic triad members. More tonal groupings result, and this, in turn, decelerates the overall rate at which the melody appears to unfold.

\section{GENERAL DISCUSSION}

As a set, the results of the two experiments reported here extend the music cognition literature in at least two different directions. First, they demonstrate that a melody's tempo can be influenced by different aspects of event structure. Some of these involve nontemporal information in the form of pitch relations. In Experiment 1, it was found that both multiple changes in pitch direction and a greater magnitude of pitch skips decelerated the apparent rate of auditory motion. In addition, Experiment 2 demonstrated that temporal dimensions other than velocity itself can influence perceptual judgments. Patterns of temporal accentuation that coincided with different levels of melodic change were judged to transpire more quickly than did those that conflicted with melodic accents and the organization of pitch relations. These effects were very robust ones that generalized not only across a significant proportion of the subjects in the experiment and different melodic instances but to different tempi as well. Although pitch and rhythm could enhance or reduce the tempo of melodies that were actually faster or slower than their standard referents, they, more interestingly, induced tempo differences when none existed.

This overall pattern of results has been suggested to stem from an overgeneralization of certain structural relations that are typically found within Western music. In most compositions, melodic phrase boundaries are accentuated by temporal changes that previous research has shown to be perceptually salient to listeners: Phrase boundaries not only provide cognitive referents for expectancy generation and selective attending purposes but for remembering as well. Given that this relationship is a relatively invariant one that appears across a wide genre of music, it eventually becomes well learned, so that listeners come to expect joint accentuation in future encounters with music. When absent, the results here suggest that people impute ritards and prolonged durations to melodic phrase boundaries. In Experiment 1, those melodies that displayed salient melodic accents were judged to be slower than those that lacked any structural markers of melodic phrasing. Similarly, the conflicting accent structures of Experiment 2 were judged to be slower than compatible ones, presumably because melodic accents that were temporally unaccented were perceived to contain temporal changes. In both cases, the subjects perceptually confirmed their expectancies by imputing temporal accents to melodic phrase boundaries.
If this imputed velocity hypothesis is a valid one, this in turn leads to a corollary set of predictions involving judged duration behavior. That is, imposing ritards and prolonged durations on melodic phrase boundaries should not only decelerate perceived tempo but also result in longer estimates of a melody's total time span. The past research on time estimation behavior suggests that this is, in fact, the case. In a study by Boltz (1991b), musical compositions displaying a greater number of contour changes were judged to be relatively longer than those displaying fewer changes, even though all tunes were actually equivalent in total duration. In addition, melodies containing incompatible accent structures, like those in the present study, have consistently been found to yield overestimates of judged duration, whereas compatible rhythms typically produce both shorter and more accurate estimates (Boltz, 1992, 1995, 1998). Although effects that are due to the magnitude of pitch skips have not been previously investigated, one would predict that these, too, will result in a lengthening of temporal experience. In sum, then, overgeneralizations of temporal/ melodic accent structures appear to influence not only tempo perception but judged duration as well.

There is a second interpretation of the present data that is not necessarily inconsistent with the imputed velocity hypothesis but, instead, attributes illusory tempo changes in music to more global principles of motion perception. That is, people may overgeneralize certain invariant relations that typically apply to visual motion to events within the auditory environment. This idea, of course, rests on the assumption that both sensory modalities share analogous dimensions of spatiotemporal structure. Several individuals, in fact, have made this argument (Julesz \& Hirsh, 1972; Kubovy, 1981; Marks, 1978 ). Within the visual modality, the spatial dimension of events is rather self-evident, in that people and other objects move over varying distances from one point to another. Similarly, pitch relations can be envisioned in an analogous fashion: They are often categorized as high or low, span different distances over successive intervals, and form a particular contour over a melody's total time span. The temporal dimensions of velocity, rhythm, and duration can also be identified in both modalities, in that auditory and visual objects move at a given rate and with a particular pattern of durational change over a total time span.

Given these analogous dimensions, it may be the case that the effects of pitch contour, interval distance, and rhythmic compatibility on musical tempo perception stem from similar phenomena within the visual environment. First, consider contour or changes in pitch direction. Just as contour accents are typically characterized by a decelerated tempo, the same is true of the trajectory for both animate and inanimate objects moving in the world around us. This, for example, can be observed in an animal running a zigzagged course over the open terrain: Before each shift in direction, the animal must reduce its overall speed in order to remain upright and maintain its bal- 
ance. A related set of circumstances may apply to effects of pitch magnitude. In the natural environment, greater distances typically take more time to traverse and, given this longer duration, are often associated with a slower velocity - a phenomenon that has been termed the kappatau effect (Cohen \& Cooper, 1962, 1963). Lastly, the effects of rhythmic (in)compatibility may also stem from more general principles of motion. Relative to joint accent structures in which melodic information unfolds in a smooth and temporally integrated fashion, incompatible accent structures display temporal and melodic information that are structurally dissociated from one another and thereby appear disjointed in nature. Similar rhythms can be identified in the visual environment, which, in turn, have been associated with tempo differences. For example, when examining the walking gait of different individuals, those whose body parts (i.e., the swinging of the legs, arms, head) move together in a coordinated fashion typically display a faster gait and are able to accelerate to a higher rate of speed than those whose various body parts are relatively uncoordinated and move out of phase from one another (Pierson, 1976).

In sum, then, this alternative account of the present data suggests that the perception of auditory motion is influenced by those principles that apply to visual motion within the natural environment. Again, this interpretation is not necessarily inconsistent with the earlier one, because, in both cases, people are overgeneralizing certain invariant relations that typically accompany velocity changes. Hence, although the relevant set of invariants varies across the two accounts, the underlying cognitive process is the same in each. In fact, given that dimensions of pitch contour, interval distance, and rhythmic compatibility can be argued to have visual analogues that influence tempo changes in the same direction as joint accent structures in music, both sets of principles may be operating together in tandem to influence people's behavior. Additional research is needed to disentangle these two hypotheses, by determining whether other types of pitch attributes result in perceived tempo changes, even though they play no role in the use of joint accent structures within musical compositions.

In addition to demonstrating that different aspects of event structure influence tempo perception, the present results also indicate that the observed effects are interactive and not independent in nature: Tempo judgments varied across the different levels of contour change and interval distance as well as rhythmic compatibility. From a cognitive perspective, this indicates that, in lieu of attending to one dimension to the exclusion of the other, the subjects simultaneously directed attending to both a melody's temporal and its nontemporal information. On the one hand, this is somewhat puzzling, because, if subjects had chosen to attend to a melody's actual tempo while ignoring the accompanying melodic information, no tempo distortions would have occurred. The question, then, is why this was the case and, more generally, what factors have led to an independent rather than an interactive relationship between temporal and nontemporal information.

To a large extent, the answer to these questions appears to involve task demands and the way that a melody's information must be used by subjects. When considering the present data relative to the past literature, interactive relationships primarily seem to arise when subjects are asked to discriminate, recognize (Boltz, 1993; Jones et al., 1982), or recall (Boltz, 1991a, 1992, 1998; Boltz \& Jones, 1986) a melody's information. In order to perform these types of tasks, it is most adaptive to attend to both a melody's temporal and its nontemporal information, because, given the prevalence of joint accent structures in Western music, these two dimensions will directly coincide and mutually reinforce one another. This, in turn, will not only highlight the melody's underlying organization and the relationships among both adjacent and nonadjacent notes but will also allow listeners to generate expectancies about the melody's future course. Hence, this type of joint attending strategy will ensure an optimal pickup of the relevant information that is needed for the task at hand. The drawback is that, in the case of incompatible accent structures, cognitive performance will be impaired. Given that temporal accents conflict with melodic ones, the melody's underlying organization will be obscured and thereby learning and remembering will decrease. In addition, the melody will appear to unfold more slowly and give rise to the types of illusory tempo changes observed here. Incompatible accent structures, however, are less common than joint ones, and so, on the average, attending to temporal and nontemporal information together will result in the highest level of performance.

Independent relationships, on the other hand, have primarily been observed when subjects are asked to make evaluative judgments of a melody's information, such as judging the degree of similarity among different tunes (Monahan \& Carterette, 1985). The cognitive demands of these types of tasks are very different from those of learning, remembering, and discrimination, because, in order to fulfill the tasks' requirements, one can opt to focus on one structural dimension alone. This may not only reduce attentional demands but actually facilitate the selection of that dimension that best distinguishes one cluster of melodies from another.

In sum, then, it is suggested that independent, as opposed to interactive, relationships among a melody's structural dimensions depend, at least in part, on the particular demands of a given task. When subjects must learn and subsequently recapitulate a melody from memory, it is most advantageous to attend to both a melody's temporal and its nontemporal information. Similarly, the tempo discrimination task of the present experiment benefits from this type of strategy, because, given the convention of joint accentuation, attending to melodic information in conjunction with a melody's tempo will ensure the pickup 
of those temporal changes that typically accompany phrase boundary locations. It is those tasks that benefit from attending to one dimension to the exclusion of the other that are most apt to result in independent relationships.

The results observed in the present set of experiments raise not only questions of a theoretical nature but also ones of generalizability. In many ways, these studies are preliminary ones that suggest several avenues for future research. There are at least three that immediately come to mind. The first is a need for converging operations and investigating the effects of pitch and rhythm in other experimental contexts. In addition to perceptual rating tasks, it would be useful to employ psychophysical techniques to determine whether the JND for tempo discrimination varies as a function of pitch relations and tempo$\mathrm{ral} /$ melodic accent structure. It may also be of interest to assess the effects of these variables on memory and examine whether certain types of musical structure lead to a more accurate remembering of tempo information than do others and whether any systematic patterns of bias emerge. A second issue involves musical expertise. The subjects in the present experiments were all nonmusicians who regularly listened to music but had no experience in musical performance. Hence, it is unclear whether results are restricted to this population or generalize to both musicians and nonmusicians alike. Perhaps musicians are less apt both to perceive tempo differences when none exist and to accelerate or decelerate the rate of actual tempo differences. This, too, remains an empirical question. Lastly, there are many other aspects of musical structure that may influence tempo perception and yet have not been previously investigated. These include the potential impact of harmony, tonal resolution within a melody, expectancy confirmations and violations, as well as a number of other variables. Relative to other subdisciplines within the field of music cognition; the influence of tempo on cognitive performance has not been well investigated and is open to a wide range of future research.

\section{REFERENCES}

Allan, L. G. (1979). The perception of time. Perception \& Psychophysics, 26, 340-354.

Benjamin, W. (1984). A theory of musical meter. Music Perception, 1 , 355-413.

BERRY, W. (1976). Structural functions in music. Englewood Cliffs, NJ: Prentice-Hall.

BLock, R. A. (1979). Time and consciousness. In G. Underwood \& R. Stevens (Eds.), Aspects of consciousness: Vol. I. Psychological issues (pp. 179-217). London: Academic Press.

Boltz, M. [G.] (1989). Rhythm and "good endings": Effects of temporal structure on tonality judgments. Perception \& Psychophysics, 46, 9-17.

BolTz, M. [G.] (1991a). Some structural determinants of melody recall. Memory \& Cognition, 19, 239-251.

Boltz, M. [G.] (1991b). Time estimation and attentional perspective. Perception \& Psychophysics, 49, 422-433.

BoLTz, M. G. (1992). The remembering of auditory event durations. Journal of Experimental Psychology: Learning, Memory, \& Cognition, 18, 938-956.

Boltz, M. G. (1993). The generation of temporal and melodic expec- tancies during musical listening. Perception \& Psychophysics, 53, 585-600.

BoLtz, M. G. (1994). Changes in internal tempo and effects on the learning and remembering of event durations. Journal of Experimental Psychology: Learning, Memory, \& Cognition, 20, 1154-1171.

BoLTZ, M. G. (1995). Effects of event structure on retrospective duration judgments. Perception \& Psychophysics, 57, 1080-1096.

BoLtz, M. G. (1998). The processing of temporal and nontemporal information in the remembering of event durations and musical structure. Journal of Experimental Psychology: Human Perception \& Performance, 24, 1087-1 104.

Boltz, M. G., \& Jones, M. R. (1986). Does rule recursion make melodies easier to reproduce? If not, what does? Cognitive Psychology, 18, 389-431.

Clarke, E. (1982). Timing in the performance of Erik Satie"s "Vexations." Acta Psychologica, 50, 1-19.

Clynes, M., \& Walker, J. (1982). Neurobiologic functions of rhythm, time and pulse in music. In J. Clynes (Ed.), Music, mind, and brain: The neuropsychology of music (pp. 171-216). New York: Plenum.

Clynes, M., \& Walker, J. (1986). Music as time's measure. Music Perception, 4, 85-120.

COHEN, J., \& COOPER, P. (1962). New phenomena in apparent duration, distance, and speed. Nature, 196, 1233-1234.

COHEN, J., \& COOPER, P. (1963). Durée, longeur, et vitesse apparentes d'un voyage. L'Année Psychologique, 1, 15-28.

Collier, G. L., \& Collier, J. L. (1994). An exploration of the use of tempo in jazz. Music Perception, 11, 219-242.

COOPER, G., \& MEYer, L. (1960). The rhythmic structure of music Chicago: University of Chicago Press.

Deutsch, D. (1980). The processing of structured and unstructured tonal sequences. Perception \& Psychophysics, 28, 381-389.

Deutsch, D., \& Feroe, J. (1981). The internal representation of pitch sequences in tonal music. Psychological Review, 88, 503-522.

Drake, C., \& BotTE, M.-C. (1993). Tempo sensitivity in auditory sequences: Evidence for a multiple-look model. Perception \& Psychophysics, 54, 277-286.

Fraisse, P. (1978). Time and rhythm perception. In E. Carterette \& W. Friedman (Eds.), Handbook of perception (Vol. 8, pp. 203-254). New York: Academic Press.

Fraisse, P. (1982). Rhythm and tempo. In D. Deutsch (Ed.), The psychology of music (pp. 149-180). New York: Academic Press.

Fraisse, P. (1984). Perception and estimation of time. Annual Review of Psychology, 35, 1-36.

FraIsse, P. (1987). Cognition of time in human activity. In G. d'Ydewalle \& W. Lens (Eds.), Cognition in human motivation and learning (pp. 233-259). Hillsdale, NJ: Erlbaum.

GERINGER, J. M. (1976). Tuning preferences in recorded orchestral music. Journal of Research in Music Education, 24, 169-176.

Geringer, J. M., \& MAdsen, C. K. (1984). Pitch and tempo discrimination in recorded orchestral music among musicians and nonmusicians. Journal of Research in Music Education, 3, 195-204.

HANDEL, S. (1993). The effect of tempo and tone duration on rhythm discrimination. Perception \& Psychophysics, 54, 370-382.

Hellstrom, A. (1985). The time-order error and its relatives: Mirrors of cognitive processes in comparing. Psychological Bulletin, 97, 35-61.

JoNES, M. R. (1981). Music as a stimulus for psychological motion: Part I. Some determinants of expectancies. Psychomusicology, 1, 34-51.

JONES, M. R. (1987a). Dynamic pattern structure in music: Recent theory and research. Perception \& Psychophysics, 41, 621-634.

Jones, M. R. (1987b). Perspectives on musical time. In A. Gabrielsson (Ed.), Action and perception in rhythm and music (pp. 153-175). Stockholm: Royal Swedish Academy of Music Press.

JoNES, M. R. \& Boltz, M. G. (1989). Dynamic attending and responses to time. Psychological Review, 96, 459-491.

Jones, M. R., Boltz, M. [G.], \& KidD, G. (1982). Controlled attending as a function of melodic and temporal context. Perception \& Psychophysics, 32, 211-218.

Julesz, B., \& Hirsh, I. J. (1972). Visual and auditory perception: An essay of comparison. In E. E. David \& P. Denes (Eds.), Human communication: $A$ unified view (pp. 283-340). New York: McGraw-Hill. 
KRAMER, J. (1982). Beginnings and endings of Western art music. Canadian University Music Review, 3, 1-14.

Kronman, U., \& SUndBerg, J. (1987). Is the musical ritard an allusion to physical motion? In A. Gabrielsson (Ed.), Action and perception in rhythm and music (pp. 57-68). Stockholm: Royal Swedish Academy of Music Press.

Kubovy, M. (1981). Concurrent-pitch segregation and the theory of indispensable attributes. In M. Kubovy \& J. R. Pomerantz (Eds.), Perceptual organization (pp. 55-98). Hillsdale, NJ: Erlbaum.

KuHN, T. L. (1974). Discrimination of modulated beat tempo by professional musicians. Journal of Research in Music Education, 22, 270-277.

KuHN, T. L. (1977). Effects of dynamics, halves of exercise, and trial sequences on tempo accuracy. Journal of Research in Music Education, 25, 222-227.

LEBLANC, A. (1981). Effects of style, tempo, and performing medium on children's music preference. Journal of Research in Music Education, 29, 143-156.

LeBlanc, A., \& MCCraRY, J. (1983). Effects of tempo on children's music preference. Journal of Research in Music Education, 31, 283294.

LERDAHL, F., \& JACKENDOFF, R. (1983). A generative theory of tonal music. Cambridge, MA: MIT Press.

Levitin, D. J., \& CoOK, P. R. (1996). Memory for musical tempo: Additional evidence that auditory memory is absolute. Perception \& Psychophysics, 58, 927-935.

MADSEN, C. K. (1979). Modulated beat discrimination among musicians and nonmusicians. Journal of Research in Music Education, 27, 57-67.

Marks, L. E. (1978). The unity of the senses. New York: Academic Press.

MARSHBURN, E., \& JONES, M. R. (1985, May). Rhythm recognition as a function of rate: Relative or absolute? Paper presented at the meeting of the Midwestern Psychological Association, Chicago.

MeYER, L. B. (1956). Emotion and meaning in music. Chicago: University of Chicago Press.

Monahan, C., \& Carterette, E. (1985). Pitch and duration as determinants of musical space. Music Perception, 3, 1-32.

Nielzen, S., \& Cesarec, Z. (1982). Aspects of tempo and perception of music in mania. Acta Psychiatrica Scandinavia, 65, 81-85.

Palmer, C., \& KRUMhanSL, C. L. (1987). Independent temporal and pitch structures in determination of musical phrases. Journal of Experimental Psychology: Human Perception \& Performance, 13, 116-126.
Pierson, K. (1976). Control of walking. Scientific American, 235, 72-86. Povel, D. (1977). Temporal structure of performed music. Some preliminary observations. Acta Psychologica, 41, 309-320.

RASCH, R. (1979). Synchronization in performed ensemble music Acoustica, 43, 121-131.

RASCH, R. (1988). Timing and synchronization in ensemble performance. In J. Sloboda (Ed.), Generative processes in music: The psychology of performance, improvisation, and composition (pp. 70-90). Oxford: Oxford University Press.

REPP, B. H. (1992). Diversity and commonality in music performance An analysis of timing microstructure in Schumann's "Traumerei." Journal of the Acoustical Society of America, 92, 2546-2568.

SCHMUCKLER, M. A. (1990). The performance of global expectations. Psychomusicology, 9, 122-147.

SCHMUCKLeR, M. A., \& Boltz, M. G. (1994). Harmonic and rhythmic influences on musical expectancy. Perception \& Psychophysics, 56, 313-325.

SteIN, J. (1977). Tempo errors and mania. American Journal of Psychiatry, 134, 454-456.

ToDD, N. (1985). A model of expressive timing in tonal music. Music Perception, 3, 33-57.

ToDD, R. E., Boltz, M. [G.], \& Jones, M. R. (1989). The Midilab auditory research system. Psychomusicology, 8, 83-96.

WANG, C. C. (1984). Effects of some aspects of rhythm on tempo perception. Journal of Research in Music Education, 32, 169-176.

WANG, C. C., \& SalzBERG, R. S. (1984). Discrimination of modulated music tempo by string students. Journal of Research in Music Education, 32, 123-131.

WAPNICK, J. (1980). The perception of musical and metronomic tempo change in musicians. Psychology of Music, 8, 3-12.

YARBROUGH, C. (1987). The effect of musical excerpts on tempo discriminations and preferences of musicians and nonmusicians. In C. K. Madsen \& C. A. Prickett (Eds.), Applications of research in music behavior (pp. 175-189). Tuscaloosa: University of Alabama Press.

YESTON, M. (1976). The stratification of musical rhythm. New Haven: Yale University Press.

(Manuscript received March 10, 1997; revision accepted for publication November 28, 1997.) 